\title{
Editorial
}

\section{Advances in oral surgery}

Oral surgery has recently become a medical specialty in France, and this specialty is common to dentists and physicians. Although several odontologists already practiced oral surgery on an exclusive basis, there was no equivalent official specialty previously. For physicians, oral surgery replaced the specialty of stomatology. Oral surgery is a double-entry specialty involving the fields of medicine and dentistry. The field of oral surgery was initially difficult to set up; however, oral surgery currently forms an essential part in the training of both dentists and physicians.

The first batch of Diplôme d'Études Spécialisées de Chirurgie Orale (DESCO) graduated in 2015. Some past residents are practicing privately, whereas some others are working at hospitals and universities. This field is still young and ripe for development. During the study course, regardless of their background, all oral surgery residents are trained in both medical and dental methods such that they can work in both oral and maxillofacial surgery departments. Hence, the residents acquire thorough knowledge via this new field. Moreover, they learn to acknowledge their fellow companions working in the other departments and to socialize with and understand each other beyond their original fields and potential prejudices that arise from a lack of knowledge regarding the other individuals and their professions. "Let us enrich ourselves with our mutual differences" quoted Paul Valéry; through their daily exchange of knowledge and mutual esteem, the alumni have succeeded in creating the Oral Surgery Corporation.

We are proud of their accomplishments!

In France, the term oral surgery was officially introduced in 2011. Surprisingly, it is still not a well-known specialty. Nevertheless, the term oral is very well anchored in the medical landscape of Anglo-Saxon countries. In France, oral surgery was previously called stomatology or buccal surgery. It is up to us now to popularize the term oral in the French mindset and its links to other specialties.

When it was newly introduced, for reasons of brevity and signage, oral surgery as a term was used to describe oral surgery and oral medicine. We also renamed our journal Buccal Medicine and Buccal Surgery to Oral Medicine and Oral Surgery. Today, our journal, which is now in English, is called The Journal of Oral Medicine and Oral Surgery (JOMOS). The shift in our journal language from French to English probably represents a sacrifice on the part of our predominantly French-speaking readers. As the President of SFCO, I apologize for this inconvenience; however, it cannot be ignored that this editorial choice has led to ample rewards in terms of the wide distribution of our journal. Moreover, the online views of the journal, with free full access on www.jomos.org, have exponentially increased since this shift, with more than 120000 downloads having taken place over the first 6 months of 2018!

The term oral surgery and oral medicine certainly defines the real focus of our specialty, which also includes oral medicine. Oral medicine is a field shared with other specialties with which we maintain excellent working relationships, the first and foremost being dermatology and anatomic pathology. These cordial working relationships led to the creation of the Groupe pour l'Étude de la Muqueuse Buccale (GEMUB) in 2017. The main purpose of this group is to gather medical practitioners with an interest in oral medicine so as to exchange and release recommendations concerning clinical practice in fields with little consensus in the management of particularly frequent conditions, such as lichen planus. It is therefore a matter of great pride for SFCO that Professor JeanChristophe Fricain, the first President of GEMUB, is an active member of our society.

My vision for oral surgery is that those involved should continue to develop a feeling of belonging to a true community. It is precisely in this spirit that in 2019 SFCO has set up a professional Workplace Forum reserved for its members. This forum includes French-speakers; however, non-French-speaking members are also welcome. The forum is a place for knowledge exchange, mutual support, communication, and reflection between practitioners of oral surgery and oral medicine. While we may always continue to meet at conferences, this user-friendly platform will allow the members to keep in touch with their community every day of the year. This couldn't get easier! If you are not yet a member of SFCO, this a good reason to join us. We wish you all the best!

Go ahead oral surgery!

Professor Jacques Henri Torres, President of SFCO 